\title{
Determination of the spontaneous polarization of wurtzite $(\mathrm{Mg}, \mathrm{Zn}) \mathrm{O}$
}

Marko Stölzel, Alexander Müller, Gabriele Benndorf, Michael Lorenz, Christian Patzig, Thomas Höche, and Marius Grundmann

Citation: Appl. Phys. Lett. 104, 192102 (2014); doi: 10.1063/1.4875919

View online: https://doi.org/10.1063/1.4875919

View Table of Contents: http://aip.scitation.org/toc/apl/104/19

Published by the American Institute of Physics

\section{Articles you may be interested in}

Spontaneous and piezoelectric polarization effects in wurtzite $\mathrm{ZnO} / \mathrm{MgZnO}$ quantum well lasers Applied Physics Letters 87, 253509 (2005); 10.1063/1.2149294

Polarity in GaN and ZnO: Theory, measurement, growth, and devices

Applied Physics Reviews 3, 041303 (2016); 10.1063/1.4963919

Investigation of high density two-dimensional electron gas in $\mathrm{Zn}$-polar $\mathrm{BeMgZnO/ZnO} \mathrm{heterostructures}$ Applied Physics Letters 111, 182101 (2017); 10.1063/1.4993853

Two dimensional electron gases induced by spontaneous and piezoelectric polarization in undoped and doped AlGaN/GaN heterostructures

Journal of Applied Physics 87, 334 (2000); 10.1063/1.371866

Two-dimensional electron gas in $\mathrm{Zn}$-polar $\mathrm{ZnMgO} / \mathrm{ZnO}$ heterostructure grown by metal-organic vapor phase epitaxy

Applied Physics Letters 97, 111908 (2010); 10.1063/1.3489101

A comprehensive review of $\mathrm{ZnO}$ materials and devices

Journal of Applied Physics 98, 041301 (2005); 10.1063/1.1992666

\section{Conference Proceedings}




\title{
Determination of the spontaneous polarization of wurtzite (Mg,Zn)O
}

\author{
Marko Stölzel, ${ }^{1, a)}$ Alexander Müller, ${ }^{1}$ Gabriele Benndorf, ${ }^{1}$ Michael Lorenz, ${ }^{1}$ \\ Christian Patzig, ${ }^{2}$ Thomas Höche, ${ }^{2}$ and Marius Grundmann ${ }^{1}$ \\ ${ }_{1}^{1}$ Institut für Experimentelle Physik II, Universität Leipzig, Linnéstr. 5, 04103 Leipzig, Germany \\ ${ }^{2}$ Fraunhofer Institute for Mechanics of Materials IWM, Center for Applied Microstructure Diagnostics, \\ Walter-Huelse-Straße 1, 06120 Halle (Saale), Germany
}

(Received 21 March 2014; accepted 28 April 2014; published online 12 May 2014)

\begin{abstract}
We report on the experimental determination of the spontaneous polarization of wurtzite$(\mathrm{Mg}, \mathrm{Zn}) \mathrm{O}$ by examination of the recombination dynamics of polar $\mathrm{ZnO} /(\mathrm{Mg}, \mathrm{Zn}) \mathrm{O}$ quantum wells (QWs). The thickness-dependent decay time of the unscreened single-exciton states inside the QWs was modeled by a self-consistent solution of Schrödinger- and Poisson-equation to deduce the total polarization across the QW for different $\mathrm{Mg}$-contents inside the barriers. By the separation of the piezoelectric components of the polarization, a linear increase in spontaneous polarization with increasing Mg-content $x$ of $P / x=(0.151 \pm 0.015) \mathrm{C} / \mathrm{m}^{2}$ was determined for $\mathrm{Mg}_{\mathrm{x}} \mathrm{Zn}_{1-\mathrm{x}} \mathrm{O}$. (C) 2014 AIP Publishing LLC. [http://dx.doi.org/10.1063/1.4875919]
\end{abstract}

The transparent semiconductor $(\mathrm{Mg}, \mathrm{Zn}) \mathrm{O}$ has prominent properties for electric (as channel material in transistors) ${ }^{1}$ as well as optoelectronic (as barrier material in $\mathrm{ZnO}$-based quantum well (QW) structures) ${ }^{2}$ applications. Wurtzite (wz)$(\mathrm{Mg}, \mathrm{Zn}) \mathrm{O}$ is a polar material exhibiting a spontaneous polarization ( $\mathrm{sp}$ ) along the $c$-axis, increasing with $\mathrm{Mg}$-content. Up to now, calculated values of the spontaneous polarization of wz-(Mg,Zn)O vary strongly, e.g., listed in detail in Ref. 3. Experimental determination is difficult to achieve due to the minor influence in optical transitions in bulk material. In contrast, the polarization or rather the change in polarization between different materials has a tremendous influence on the transition energy as well as decay time of excitons in QW structures due to the quantum-confined Stark effect (QCSE). The difference in polarization between the barrier and well material leads to an internal electric field across the QW. For well widths larger than the exciton Bohr radius $(\sim 2 \mathrm{~nm}$ in $\mathrm{ZnO})$, the internal electric field separates electrons and holes inside the QW. This leads to a decrease of the transition energy (triangular potential for electrons and holes) and large increase of the decay time (from hundreds of picoseconds up to milliseconds). In this paper, we investigate the building blocks of optoelectronic devices, namely, $\mathrm{ZnO} /(\mathrm{Mg}, \mathrm{Zn}) \mathrm{O}$ QWs, differing in well width, $\mathrm{Mg}$-content in the barriers to experimentally determine the spontaneous polarization of wz-( $\mathrm{Mg}, \mathrm{Zn}) \mathrm{O}$.

For pseudomorphic growth of quantum well structures, at least one of the materials is strained leading to an additional piezoelectric polarization $(\mathrm{pz})$. The resulting electric field across the $\mathrm{QW} E=\Delta P_{\mathrm{QW}} / \epsilon$ is determined by the difference in total polarization between the layers $\Delta P_{\mathrm{QW}}$ and the static dielectric constant $\epsilon . \Delta P_{\mathrm{QW}}$ can be expressed as the sum of the differences of spontaneous and piezoelectric polarizations between the layers $\Delta P_{\mathrm{QW}}=\Delta P_{\mathrm{sp}}+\Delta P_{\mathrm{pz}}$.

The single $\mathrm{QW}$ structures investigated in this study were grown by pulsed laser deposition (PLD). ${ }^{4}$ The $(\mathrm{Mg}, \mathrm{Zn}) \mathrm{O}$ layers were deposited either directly or with a $\mathrm{ZnO}$ buffer layer on $a$-plane sapphire as well as on $\mathrm{ZnO}$ substrates by

${ }^{\text {a)} E l e c t r o n i c ~ m a i l: ~ s t o e l z e l @ p h y s i k . u n i-l e i p z i g . d e ~}$ ablation of sintered ceramic targets with the $248 \mathrm{~nm}$ line of a $\mathrm{KrF}$ laser and a laser fluence of $1.8 \mathrm{~J} / \mathrm{cm}^{2}{ }^{5}$ The samples were grown in an oxygen ambient (0.004 millibars) at a substrate temperature of $650{ }^{\circ} \mathrm{C}$. Pseudomorphic growth of $(\mathrm{Mg}, \mathrm{Zn}) \mathrm{O}$ on $\mathrm{ZnO}$ substrates and $\mathrm{ZnO}$ buffer layers has been verified by $\mathrm{x}$-ray diffraction. ${ }^{6}$ In contrast, direct growth of $(\mathrm{Mg}, \mathrm{Zn}) \mathrm{O}$ on sapphire leads to relaxed barrier layers with a strained $\mathrm{ZnO}$ QW. Hall-effect measurements on comparable samples show a n-type background doping in the region of $10^{17} \mathrm{~cm}^{-3}$. To investigate an identical barrier composition for different well widths, a thickness gradient was introduced (described explicitly in Ref. 5). The gradient is such that the thickness changes at most by one mono layer within the excitation area $(\sim 100 \mu \mathrm{m})$ of the photoluminescence (PL) measurements. Such wedge-shaped as well as planar samples have been investigated. The QW thickness was calculated from the number of pulses applied to the target assuming a constant growth rate. The growth rate itself was determined for selected samples by X-ray reflectometry and Z-contrast scanning transmission electron microscopy (STEM) as shown for one sample in Fig. 1. In this paper, we focus on time-resolved (TR-) PL spectroscopy measurements. The PL was excited by the 200 fs pulses of a frequency doubled Ti:sapphire laser. The wavelength has been tuned beneath the bandgap of the $(\mathrm{Mg}, \mathrm{Zn}) \mathrm{O}$ layers to exclude effects on the carrier dynamics inside the QW. ${ }^{7}$ The TR-PL was detected by time-correlated single-photon counting with a time resolution down to 20 ps. An acousto-optical modulator was used to decrease the repetition rate of the laser from $76 \mathrm{MHz}$. The PL experiments were performed in a He-bath cryostat with a sample temperature of $2 \mathrm{~K}$.

PL spectra of three QWs with the same well width but differing in $\mathrm{Mg}$-content in the barrier layers are depicted in Fig. 2. A strong redshift of the QW transition energy is visible due to the increasing QCSE with enlarged Mg-content in the barriers. The luminescence originates from partly screened free excitons inside QWs. ${ }^{9,15}$ Carrying out luminescence investigations on these samples, screening effects have to be taken into account even at moderate to low excitation densities $\left(\sim 1 \mathrm{~W} / \mathrm{cm}^{2}\right) .8,9$ The PL peak position of the unscreened 


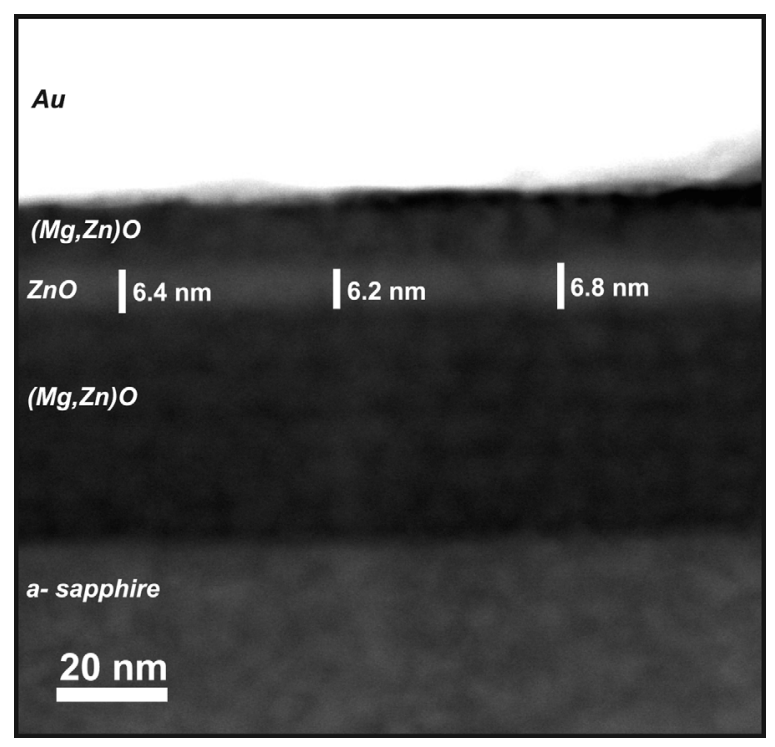

FIG. 1. Z-contrast STEM image of a $\mathrm{ZnO} /(\mathrm{Mg}, \mathrm{Zn}) \mathrm{O}$ QW in [0001] direction with a $\mathrm{Mg}$ content of $22 \%$ in the barrier layers. The thickness of the QW was measured on several positions as indicated.

exciton state $E_{0}$ inside the QW is very hard to determine with a significant signal-to-noise ratio (no analytical description or model exists for the dependence of the transition energy on screening). In contrast, we previously described a reliable method to precisely determine the decay time $\tau_{0}$ of the unscreened exciton state by analyzing the energetic dependence of the decay time using a extended relaxation model. ${ }^{9}$ Therefore, the non-exponential transients measured at different energetic positions across the QW luminescence peak are modeled by a stretched exponential decay function to determine the average decay time $\tau(E)$ in dependence on the emission energy. Afterwards, $\tau(E)$ is fitted by the extended relaxation model to determine $\tau_{0}$ (for details see Ref. 9).

In the following, we will first present data for QW samples deposited directly on $a$-plane sapphire substrates. The decay time of the unscreened single-exciton state in dependence on well width of these samples is depicted in Fig. 3 for

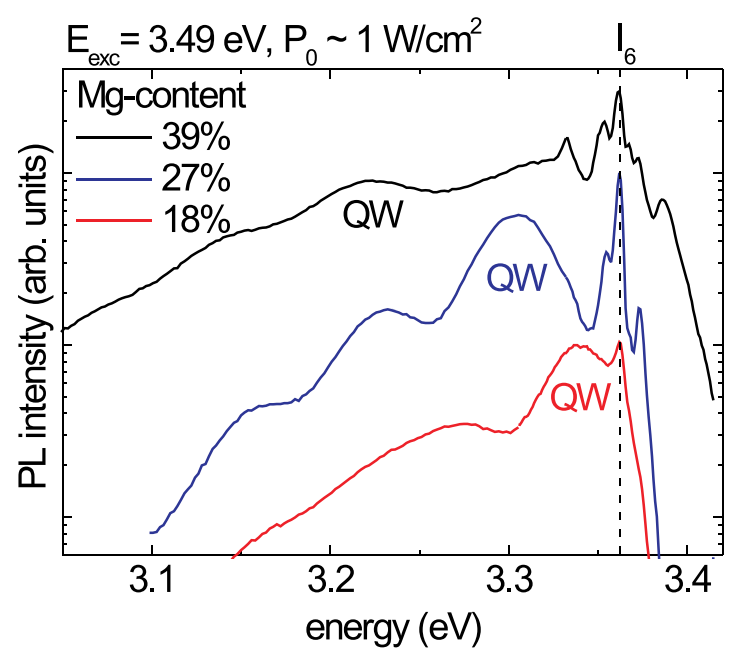

FIG. 2. PL spectra of three $5.8 \mathrm{~nm}$ QW structures deposited on $\mathrm{ZnO}$ buffer layer on $a$-plane sapphire differing in $\mathrm{Mg}$-content in the barriers. The peaks around $3.37 \mathrm{eV}$ stem from donor bound excitons out of the buffer layer. The strongest transition $\left(\mathrm{I}_{6}\right)$ has been indicated by a dashed line.

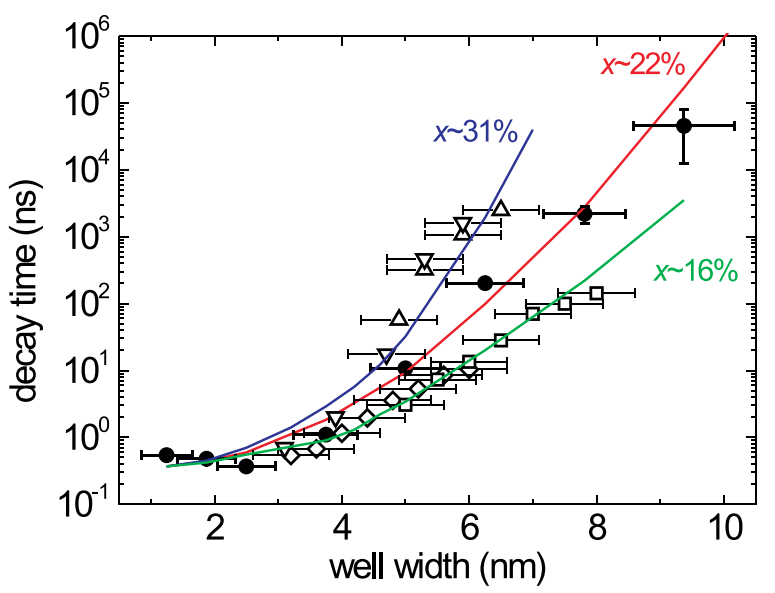

FIG. 3. Dependence of the single-exciton decay time on the QW width for $\mathrm{ZnO} /(\mathrm{Mg}, \mathrm{Zn}) \mathrm{O}-\mathrm{QWs}$ deposited directly on $a$-plane sapphire with three different $\mathrm{Mg}$-contents $x$ in the barriers. Dots represent planar samples with $x=22 \%$. Triangles $(x=31 \%)$ and squares $(x=16 \%)$ represent wedgeshaped samples. The solid lines represent the simulated data for the indicated $\mathrm{Mg}$-content in the barrier layers.

three different Mg-contents $x$ in the barrier layers. Clearly visible is an enormous increase of the decay time above $3 \mathrm{~nm}$ well width due to the QCSE (the decay times of QWs without the QCSE vary only little with well width ${ }^{7}$ ). In dependence of the Mg-content in the barriers, the slope differs due to the different internal electric field strength. The total polarization $\Delta P_{\mathrm{QW}}$ across the $\mathrm{QW}$ was estimated from numerical calculations.

The wave-functions of the uncorrelated electrons and holes were calculated by solving the Schrödinger and Poisson equation self-consistently. ${ }^{10}$ The exciton binding energy as well as exciton wave-function have been calculated in the envelope function approximation by a variational approach. ${ }^{11}$ The two-body exciton wave-function is written as

$$
\Phi=\Phi_{\mathrm{e}}\left(z_{\mathrm{e}}\right) \Phi_{\mathrm{h}}\left(z_{\mathrm{h}}\right) \Phi_{\mathrm{r}}\left(r_{\perp},\left|z_{\mathrm{e}}-z_{\mathrm{h}}\right|\right),
$$

with the uncorrelated electron and hole wave-function $\Phi_{\mathrm{e} / \mathrm{h}}$. $\Phi_{\mathrm{r}}$ symbolizes the in-plane exciton wave-function

$$
\Phi_{\mathrm{r}}=\exp \left(-\frac{\sqrt{r_{\perp}^{2}+\zeta^{2}\left|z_{\mathrm{e}}-z_{\mathrm{h}}\right|^{2}}}{\lambda}\right)
$$

depending on the in-plane elongation $r_{\perp}$ with a dimensionless parameter $0 \leq \zeta \leq 1$ describing the dimensionality of the exciton, and the exciton Bohr radius $\lambda$ as proposed in Ref. 12. Only the lowest electron and heavy hole eigenstates of the uncorrelated system were regarded for the calculation. The energy of the exciton was minimized by variation of $\zeta$ and $\lambda$ to obtain the oscillator strength and the transition energy.

The following parameters were used: $m_{\mathrm{e}}=0.24 m_{0}$ and $m_{\mathrm{h}}=0.91 m_{0}$ for electron and hole on-axis effective masses, in both well and barrier material, $\mathrm{E}_{\mathrm{c}} / \mathrm{E}_{\mathrm{v}}=90 / 10$ as band discontinuity, a net background doping of $10^{17} \mathrm{~cm}^{-3}$ donors and $\epsilon_{\mathrm{b}}=6.4$ for the background dielectric constant. ${ }^{9,13-15}$

The difference in total polarization between well and barrier material was simulated for each Mg-content. The solid 


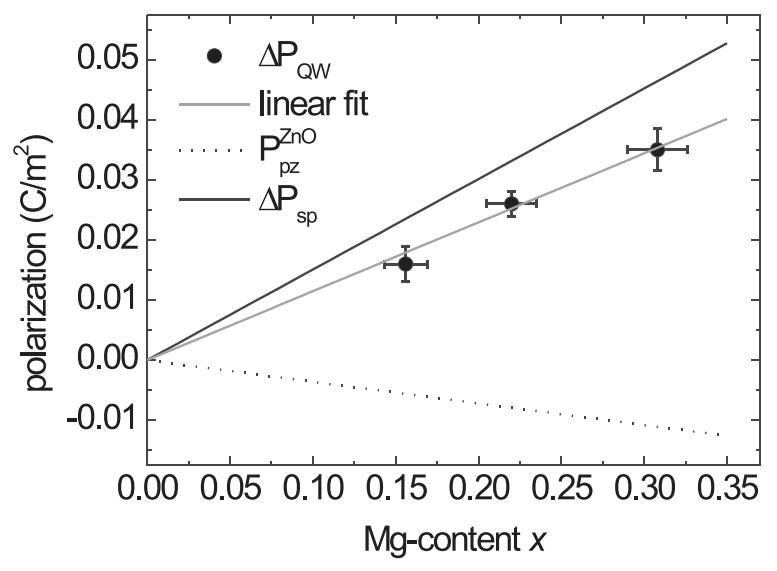

FIG. 4. Linear fit (grey line) of the experimental determined total polarization difference $\Delta P_{\mathrm{QW}}$ (dots) between $\mathrm{QW}$ and barrier layers for pseudomorph grown $\mathrm{ZnO}$ on relaxed $(\mathrm{Mg}, \mathrm{Zn}) \mathrm{O}$ barrier layers deposited on $a$-plane sapphire. The piezoelectric polarization (dotted line) has been calculated for the $\mathrm{ZnO} \mathrm{QW}$. For the relaxed barrier layers, $P_{\mathrm{pz}}$ equals zero. The black line represents the determined difference of the spontaneous polarization between $\mathrm{ZnO}$ and $(\mathrm{Mg}, \mathrm{Zn}) \mathrm{O}$ in dependence of the Mg-content $x$.

lines in Fig. 3 represent the best fit of the resulting dependence of the inverse oscillator strength (normalized to the decay at small well widths) to the experimental data. The dots in Fig. 4 show the experimentally determined total polarizations across the $\mathrm{QW}$ in dependence on the Mg-content in the barriers. Clearly visible is an increase of $\Delta P_{\mathrm{QW}}$ with increasing $\mathrm{Mg}$ content. A linear fit gives $\Delta P_{\mathrm{QW}} / x=(0.115 \pm 0.015) \mathrm{C} / \mathrm{m}^{2}$.

The QWs, described above, were deposited directly on $a$-plane sapphire leading to relaxed and thus unstrained barrier layers. The piezoelectric polarization thereby equals zero. Therefore, the total polarization across the QW consists of the difference of the spontaneous polarization of the two materials and the piezoelectric polarization of the pseudomorph grown $\mathrm{ZnO} \mathrm{QW}$. This piezoelectric component can be calculated via

$$
P_{\mathrm{pz}}=2 \frac{a_{\mathrm{s}}-a_{\mathrm{e}}}{a_{\mathrm{e}}}\left(e_{31}-e_{33} \frac{C_{13}}{C_{33}}\right),
$$

where $\mathrm{a}_{\mathrm{s}}$ and $\mathrm{a}_{\mathrm{e}}$ represent the lattice constants of the unstrained bottom layer and the pseudomorphically grown epilayer, respectively. ${ }^{16}$ With the elastic compliance constants ${ }^{17}$ $\mathrm{C}_{13}=118 \mathrm{GPa}$ and $\mathrm{C}_{33}=211 \mathrm{GPa}$ and coefficients of the piezoelectric tensor ${ }^{18} \mathrm{e}_{31}=-0.51 \mathrm{C} / \mathrm{m}^{2}$ and $\mathrm{e}_{33}=0.89 \mathrm{C} / \mathrm{m}^{2}$, the piezoelectric polarization of the tensile strained QW layer was calculated and is depicted as a dotted line in Fig. 4. For the change of the in-plane lattice constant of the $\mathrm{ZnO}-\mathrm{QW}$ with the Mg-content of the relaxed bottom layer, we used a dependence of $a(x)(\AA)=3.244+0.058 \times x$ determined by x-ray diffraction for our samples. The piezoelectric polarization of the strained $\mathrm{ZnO}-\mathrm{QW}$ counteracts the resulting difference in spontaneous polarization reducing the internal electric field across the QW.

Subtracting the piezoelectric polarization of the $\mathrm{ZnO}$ $\mathrm{QW}$ from $\Delta P_{\mathrm{QW}}(x)$ yields the difference of the spontaneous polarization at the interface between $\mathrm{QW}$ and barrier $\Delta P_{\mathrm{sp}}=P_{\mathrm{sp}}^{\mathrm{MgZnO}}-P_{\mathrm{sp}}^{\mathrm{ZnO}}$. Using $P_{\mathrm{sp}}^{\mathrm{ZnO}}=0.057 \mathrm{C} / \mathrm{m}^{2}$ (Ref. $18)$, we find the spontaneous polarization of $\mathrm{wz}-(\mathrm{Mg}, \mathrm{Zn}) \mathrm{O}$ $P_{\mathrm{sp}}^{\mathrm{MgZnO}}=(0.057+(0.151 \pm 0.015) \times x) \mathrm{C} / \mathrm{m}^{2}$. Compared to theoretical calculations in literature the slope is about a factor five larger. Via $a b$-initio calculations based on density functional theory $\left(0.027 \mathrm{C} / \mathrm{m}^{2} \times x\right)^{3}$ or pseudo-potential selfinteraction correction method $\left(0.038 \mathrm{C} / \mathrm{m}^{2} \times x\right)$, ${ }^{19}$ the enlargement of the spontaneous polarization with $\mathrm{Mg}$-content was significantly smaller than our experimental results.

The calculated piezoelectric polarization is only valid for samples with relaxed $(\mathrm{Mg}, \mathrm{Zn}) \mathrm{O}$ barrier layers and a pseudomorphically grown $\mathrm{ZnO} \mathrm{QW}$. Depositing the QW structure to a $\mathrm{ZnO}$ buffer layer or $\mathrm{ZnO}$ substrate one expects strained barrier layers (assuming pseudomorphic growth) and a relaxed $\mathrm{ZnO} \mathrm{QW}$ layer. For this particular case, $P_{\mathrm{pz}}^{\mathrm{ZnO}}$ should equal zero. The likewise determined total polarizations of the $\mathrm{QW}$ structures are $\Delta P_{\mathrm{QW}}^{\text {buffer }} / x=(0.161 \pm 0.021) \mathrm{C} / \mathrm{m}^{2}$ for samples on a relaxed $\mathrm{ZnO}$ buffer layer and $\Delta P_{\mathrm{QW}}^{\text {substrate }} / x$ $=(0.167 \pm 0.020) \mathrm{C} / \mathrm{m}^{2}$ for samples deposited on $\mathrm{ZnO}$ substrate material. The respective data are shown in Fig. 5. The slight difference of the values may be due to different lattice constants of $\mathrm{ZnO}$ substrate and $\mathrm{ZnO}$ buffer layer on sapphire. ${ }^{20}$ Samples on $\mathrm{ZnO}$ show in total a larger polarization and therewith internal electric field across the QW than the samples deposited directly to the $a$-plane sapphire substrate consistent with prior investigations. ${ }^{9}$ We find that the resulting field across the QW is increased for compressively strained $(\mathrm{Mg}, \mathrm{Zn}) \mathrm{O}$ layers and decreased for a tensile strain of the $\mathrm{ZnO} \mathrm{QW}$ layer.

In summary, we have studied the well width dependence of the decay time of $\mathrm{ZnO} /(\mathrm{Mg}, \mathrm{Zn}) \mathrm{O}$ QW structures for different $\mathrm{Mg}$ contents in the barrier layers as well as different substrates. The total difference in polarization between the materials has been obtained by numerical calculations. Therewith, we determined the spontaneous polarization of wz- $(\mathrm{Mg}, \mathrm{Zn}) \mathrm{O}$ to $P_{\mathrm{sp}}=(0.057+(0.151 \pm 0.015) \times x) \mathrm{C} / \mathrm{m}^{2}$. We emphasize that this contribution represents a first step towards device design with structures where the piezoelectric polarization counteracts the spontaneous component in a way that the electric field across the QW is decreased specifically. Therefore, the elastic compliance constants and the piezoelectric tensor of the compound $(\mathrm{Mg}, \mathrm{Zn}) \mathrm{O}$ are to be determined.

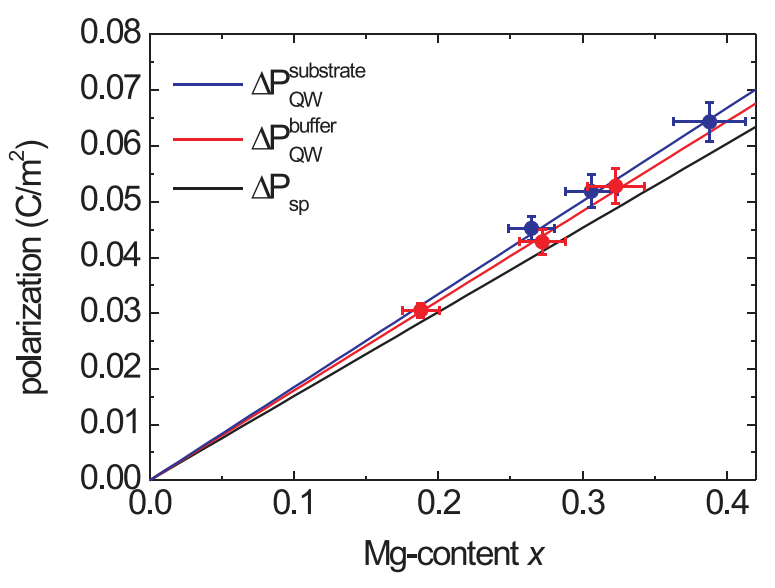

FIG. 5. Linear fits of the experimentally determined total polarization differences $\Delta P_{\mathrm{QW}}$ (dots) between $\mathrm{QW}$ and barrier layers for pseudomorphically grown ( $\mathrm{Mg}, \mathrm{Zn}) \mathrm{O}$ barrier layers on $\mathrm{ZnO}$ substrate (blue) and on relaxed $\mathrm{ZnO}$ buffer layer (red) in dependence of the Mg-content $x$. The values are compared to the determined spontaneous polarization difference $\Delta P_{\mathrm{sp}}$ between the materials (black). 
This work has been funded by the European Union and the Free State of Saxony through the European Social Fund. The authors also gratefully acknowledge the financial support by the Leipzig School of Natural Sciences BuildMoNa (GS 185) and the Deutsche Forschungsgemeinschaft in the framework of Sonderforschungsbereich SFB 762 "Functionality of oxide interfaces." In addition, we thank Gabriele Ramm for PLD target preparation and Holger Hochmuth as well as Matthias Brandt for sample growth.

${ }^{1}$ H. Frenzel, A. Lajn, and M. Grundmann, Physica Status Solidi RRL 7, 605 (2013).

${ }^{2}$ T. Makino, Y. Segawa, M. Kawasaki, A. Ohtomo, R. Shiroki, K. Tamura, T. Yasuda, and H. Koinuma, Appl. Phys. Lett. 78, 1237 (2001).

${ }^{3}$ S.-H. Jang and S. F. Chichibu, J. Appl. Phys. 112, 073503 (2012).

${ }^{4} \mathrm{M}$. Lorenz, in Transparent Conductive Zinc Oxide. Basics and Applications in Thin Film Solar Cells, Springer Series in Materials Science Vol. 104, edited by K. Ellmer (Springer, Berlin, 2007), Chap. 7, pp. 303-357.

${ }^{5}$ M. Brandt, M. Lange, M. Stölzel, A. Müller, G. Benndorf, J. Zippel, J. Lenzner, M. Lorenz, and M. Grundmann, Appl. Phys. Lett. 97, 052101 (2010).

${ }^{6}$ M. Brandt, H. von Wenckstern, M. Stölzel, H. Hochmuth, M. Lorenz, and M. Grundmann, Semicond. Sci. Technol. 26, 014040 (2011).
${ }^{7}$ J. Zippel, S. Heitsch, M. Stölzel, A. Müller, H. von Wenckstern, G. Benndorf, M. Lorenz, H. Hochmuth, and M. Grundmann, J. Lumin. 130, 520 (2010).

${ }^{8}$ C. Hall, L. Dao, K. Koike, S. Sasa, H. H. Tan, M. Inoue, M. Yano, P. Hannaford, C. Jagadish, and J. A. Davis, Phys. Rev. B 80, 235316 (2009). ${ }^{9}$ M. Stölzel, A. Müller, G. Benndorf, M. Brandt, M. Lorenz, and M. Grundmann, Phys. Rev. B 88, 045315 (2013).

${ }^{10}$ I. Tan, G. Snider, L. Chang, and E. Hu, J. Appl. Phys. 68, 4071 (1990).

${ }^{11} \mathrm{P}$. Harrison, Quantum Wells, Wires and Dots: Theoretical and Computational Physics of Semiconductor Nanostructures, 3rd ed. (Wiley, 1999).

${ }^{12}$ M. Grundmann and D. Bimberg, Phys. Rev. B 38, 13486 (1988).

${ }^{13}$ G. Coli and K. K. Bajaj, Appl. Phys. Lett. 78, 2861 (2001).

${ }^{14}$ T. Bretagnon, P. Lefebvre, T. Guillet, T. Taliercio, B. Gil, and C. Morhain, Appl. Phys. Lett. 90, 201912 (2007).

${ }^{15}$ M. Stölzel, J. Kupper, M. Brandt, A. Müller, G. Benndorf, M. Lorenz, and M. Grundmann, J. Appl. Phys 111, 063701 (2012).

${ }^{16}$ A. Reale, G. Massari, A. D. Carlo, P. Lugli, A. Vinattieri, D. Alderighi, M. Colocci, F. Semond, N. Grandjean, and J. Massies, J. Appl. Phys. 93, 400 (2003).

${ }^{17}$ G. Carlotti, D. Fioretto, G. Socino, and E. Verona, J. Phys. Cond. Matter 7,9147 (1995).

${ }^{18}$ F. Bernardini, V. Fiorentini, and D. Vanderbilt, Phys. Rev. B 56, R10024 (1997).

${ }^{19} \mathrm{P}$. Gopal and N. Spaldin, J. Electron. Mater. 35, 538 (2006).

${ }^{20}$ A. Ohtomo, M. Kawasaki, T. Koida, K. Masubuchi, H. Koinuma, Y. Sakurai, Y. Yoshida, T. Yasuda, and Y. Segawa, Appl. Phys. Lett. 72, 2466 (1998). 